\title{
AVALIAÇÃO AUDIOLÓGICA EM CAMINHONEIROS
}

Patrícia Arruda de Souza Alcarás, Nayara Gisele de Aguiar Menezes, Meire Aparecida Judai

Universidade do Oeste Paulista - UNOESTE, Curso de Fonoaudiologia, Presidente Prudente, SP. e-mail: parruda@unoeste.br

\section{RESUMO}

O estudo objetivou analisar os achados audiológicos em motoristas de caminhão, bem como analisar os sintomas auditivos e extra-auditivos presentes nesta população. A amostra foi composta por nove motoristas de caminhão do sexo masculino. A coleta dos dados deu-se pela entrevista fonoaudiológica e avaliação audiológica básica. Os resultados revelaram que a presença de zumbido, a dificuldade para entender a conversação e o incômodo a sons intensos foram os sintomas auditivos mais relatados. Já os extra-auditivos, o nervosismo foi o mais relatado. Quanto aos achados audiológicos, $88,88 \%$ apresentaram média tritonal dentro dos padrões da normalidade. Entretanto, observou-se queda do limiar auditivo nas frequências de 3000, 4000 e $6000 \mathrm{~Hz}$, sugerindo perda auditiva por exposição a ruído. Mediante aos achados, acredita-se ser necessário desenvolver ações de prevenção, visando melhores condições de trabalho nesta classe de trabalhadores.

Palavras-chave: audição, perda auditiva, motoristas.

\section{HEARING AVALIATION ON TRUCK DRIVERS}

\section{ABSTRACT}

The study aimed to analyze the audiological findings in truck drivers, as well as analyzing the hearing and extra-auditory symptoms present in this population. The sample consisted of nine male truck drivers. Data collection took place by speech therapist interview and audiological basic evaluation. The results revealed that the presence of ringing, a difficulty in understanding conversation and the discomfort to loud sounds were the most reported auditory symptoms. As for the extra-auditory, the nervousness was the most reported. As for audiological findings, $88.88 \%$ showed an average tritonal within normal standards. However, it can be observed fall of the auditory threshold in the frequencies of 3000, 4000 and $6000 \mathrm{~Hz}$, suggesting hearing loss from exposure to noise. By the findings, it is believed to be necessary to develop preventive measures, aimed at better working conditions in this class of workers.

Keywords: hearing, hearing loss, drivers

\section{INTRODUÇÃO}

Nos últimos 50 anos, houve um grande crescimento da produção de serviços no país, o que favoreceu o aumento da circulação de veículos automotores ${ }^{1}$. Sendo os caminhoneiros considerados um dos principais agentes do setor de transporte do nosso país, que movimenta ativamente o sistema econômico do Brasil, esta população está cada vez mais exposta a diversos riscos, como condições inadequadas de serviço, jornada extensa de trabalho, violências, acidentes de trânsito, entre outros.

Com intuito em verificar o estilo de vida, a saúde e o trabalho de motoristas de caminhão, autores relaram que as condições de vida e de trabalho não são favoráveis à saúde do caminhoneiro, e nessa perspectiva, faz-se necessário ações que previnam e promovam a saúde desta categoria de trabalhadores ${ }^{2}$.

O ruído no trafego é um dos principais causadores de poluição sonora. Estudos têm sido descritos com o propósito de verificar o comprometimento da saúde em motoristas mediante exposição ao ruído, visto que a atuação dessa classe de trabalhadores é de tamanha responsabilidade, os quais são responsáveis pelo cuidado e segurança de seus veículos, cargas valiosas e muitas vezes tóxicas, além do cuidado com a sua própria saúde, sendo desafiados a cada dia em suas jornadas de trabalho ${ }^{3}$.

Além disso, autores relataram que o ruído, o calor continuo dentro da cabine, a 
conformação da poltrona que nem sempre está anatomicamente correta, e a permanência em uma mesma posição frente ao volante demanda desses profissionais uma intensa atividade física e mental, o que pode comprometer a saúde em geral $^{3}$.

A atuação dos caminhoneiros pode ser de forma autônoma, prestando serviços de fretes ou contratados. Os motoristas de caminhão que trabalham de forma autônoma, na maioria das vezes não se preocupam com a saúde e não são submetidos periodicamente à avaliação ocupacional. Já os motoristas de caminhão que trabalham com carteira assinada, por ser obrigatório por lei são submetidos a exames periódicos com intuito de detectar possíveis alterações na saúde de forma precoce.

Em estudo realizado no Canadá com mais de 400 medições de níveis de pressão sonora em motoristas de caminhões expostos ao ruído ambiental, em uma jornada de 8 horas de trabalho, foi observado que a exposição excedeu o limite permitido por lei que garante a saúde auditiva destes caminhoneiros, o que pode elevar os riscos à saúde auditiva desta classe de trabalhadores ${ }^{4}$.

Entre as diversas doenças de origem ocupacional, os motoristas de caminhão podem desenvolver perdas auditivas, visto que o ruído do motor dos caminhões é elevado e nocivo à saúde auditiva. A Perda Auditiva Induzida por Ruído (PAIR) caracteriza-se por uma diminuição da acuidade auditiva pelo tempo de exposição ao ruído, cujo aparecimento ocorre, principalmente, após os cinco anos de atividade ocupacional em ambientes ruidosos, sendo de caráter irreversível, do tipo neurossesorial, e com acometimento inicial nas frequências mais altas da audição ${ }^{5}$.

Por esse motivo faz-se necessário acompanhamento periódico da audição nessa população, pois métodos de proteção, conscientização dos próprios caminhoneiros quantos aos males provocados pela exposição contínua ao ruído poderão favorecer na diminuição da ocorrência de perdas auditivas nessa população.

Para tanto, estudos envolvendo essa população favorecerá o conhecimento sobre os efeitos nocivos à audição dos caminhoneiros, uma vez que a literatura carece sobre essa demanda e com isso, será possível conscientizar essa população sobre 0 uso de métodos preventivos à saúde auditiva, bem como a realização de exames auditivos periodicamente.

Assim, o presente estudo teve por objetivo analisar os achados audiológicos em motoristas de caminhão, bem como analisar os sintomas auditivos e extra-auditivos presentes nesta população.

\section{MÉTODOS}

O presente estudo de campo, do tipo transversal e descritivo, foi aprovado pelo Comitê de Ética em Pesquisa (CEP-35008114.0.0000.551) da Universidade do Oeste Paulista.

A amostra foi composta por nove motoristas de caminhão, do sexo masculino, entre a faixa etária de 20 a 58 anos de idade, sendo estes usuários dos serviços prestados pela empresa de Serviço Social do Transporte Serviço Nacional de Aprendizagem do Transporte (SEST-SENAT), o qual o responsável pelo local aonde a amostra foi recrutada concordou em dispor dos seus usuários para realização do estudo. Além disso, a amostra também contou com a participação de motoristas autônomos.

Foram incluídos na amostra somente os motoristas de caminhão que apresentaram atividade profissional exclusivamente na área, ou seja, que fossem apenas motoristas de caminhão, com carga horária diária de atividade profissional igual ou superior a 8 horas. Foram incluídos aqueles que aceitaram e assinaram um Termo de Consentimento Livre e Esclarecido - TCLE.

Após a seleção da amostra, os participantes foram solicitados a comparecer no ambulatório de audiologia do Curso de Fonoaudiologia de uma Instituição de Ensino Superior (IES) para a coleta dos dados, que foi composta pela entrevista fonoaudiológica e avaliação audiológica básica.

A entrevista fonoaudiológica foi realizada por intermédio da aplicação do roteiro de entrevista utilizado no ambulatório de audiologia, com intuito de conhecer os sintomas auditivos e extra-auditivos apresentados pelos motoristas de caminhão.

Em seguida, foi realizada avaliação audiológica básica, composta pela pesquisa da audiometria tonal, vocal e imitanciometria.

Ambos os testes, audiometria tonal liminar e vocal foram realizados por intermédio do audiômetro Unity PC/Siemens, com os fones TDH-29, em cabine tratada acusticamente. Já a imitanciometria foi realizada com o 
imitanciômetro da marca Interacoustic e modelo AT235.

Ambos os equipamentos estavam devidamente calibrados e nenhum procedimento foi invasivo ou causou desconforto ao participante do estudo.

Além disso, antes da realização da avaliação audiológica básica, ressalta-se que todos os participantes foram submetidos à inspeção do canal auditivo externo com intuito de verificar a sua integridade, visto que se houvesse presença de rolha de cera ou corpo estranho impossibilitaria a realização da avaliação audiológica básica. Tal inspeção foi realizada por intermédio do otoscópio da marca Heidji.

Os resultados obtidos nos testes audiológicos foram comparados com a literatura 6 .

Após a coleta dos dados de cada participante envolvido na pesquisa foi emitido o parecer dos achados, oralmente e por escrito, com a entrega dos resultados de todos os exames realizados.

Os motoristas de caminhão que apresentaram alterações nos achados audiológicos foram encaminhados ao médico otorrinolaringologista para parecer médico.

Além disso, receberam orientações sobre medidas de proteção sonora, além de explicações dos malefícios que o ruído provoca no organismo como um todo.

Os dados foram analisados por meio de estatística descritiva. As comparações entre orelha direita e esquerda para o perfil auditivo dos participantes foi realizada por meio de teste $G$ ou exato de Fisher, de acordo com a necessidade. A comparação dos limites tonais auditivos das orelhas direita e esquerda dos participantes foi realizada por meio do teste de Wilcoxon. Os dados foram analisados no programa BioEstat 5.3 utilizando nível de significância de 5\%.

\section{RESULTADOS}

O presente estudo foi constituído por nove motoristas de caminhão, sendo todos do sexo masculino, entre a faixa etária de 20 a 58 anos de idade (43,33 $\pm 12,55$ anos).

O tempo de atividade profissional variou entre 5 a 37 anos $(17,67 \pm 9,76$ anos), estando todos expostos ao ruído produzido pelo motor do caminhão, no mínimo 8 horas diárias.

Em relação às atividades ocupacionais pregressas, todos referiram ter trabalhado em ambientes ruidosos, sendo que quatro $(44,44 \%)$ participantes já exerceram prática profissional como operador de máquinas, quatro $(44,44 \%)$ referiram ter trabalhado em oficinas mecânicas e um $(11,11 \%)$ participante já exerceu a profissão de tratorista.

No que se refere às queixas auditivas, cinco $(55,56 \%)$ participantes referiram não apresentar. A Figura 1 mostra os principais sintomas auditivos relatados pelos participantes do estudo.

Em relação às doenças pregressas, a Figura 2 mostra as principais doenças apresentadas pelos motoristas de caminhão.

Quanto ao uso de medicamentos, seis $(66,67 \%)$ participantes referiram fazer uso habitual de medicação, sendo que quatro $(44,44 \%)$ participantes referiram fazer uso de medicamentos para controle da pressão arterial, um $(11,11 \%)$ participante referiu uso contínuo de "Fenobarbital" e um (11,11\%) referiu uso constante de medicação para problemas estomacais.

Em relação ao histórico de cirurgia, cinco $(55,56 \%)$ referiram já terem se submetido há pelo menos um procedimento cirúrgico, sendo: vasectomia, retirada do apêndice e da vesícula, correção ortopédica por fratura na perna, retirada de tumor no braço e hérnia.

Quanto ao uso de álcool e fumo, seis $(66,67 \%)$ referiram fazer uso de tabaco e dois $(22,22 \%)$ referiram fazer uso de álcool.

Em relação aos sintomas extra-auditivos, o nervosismo foi o sintoma mais relatado pelos participantes do estudo, como mostra a Figura 3. 


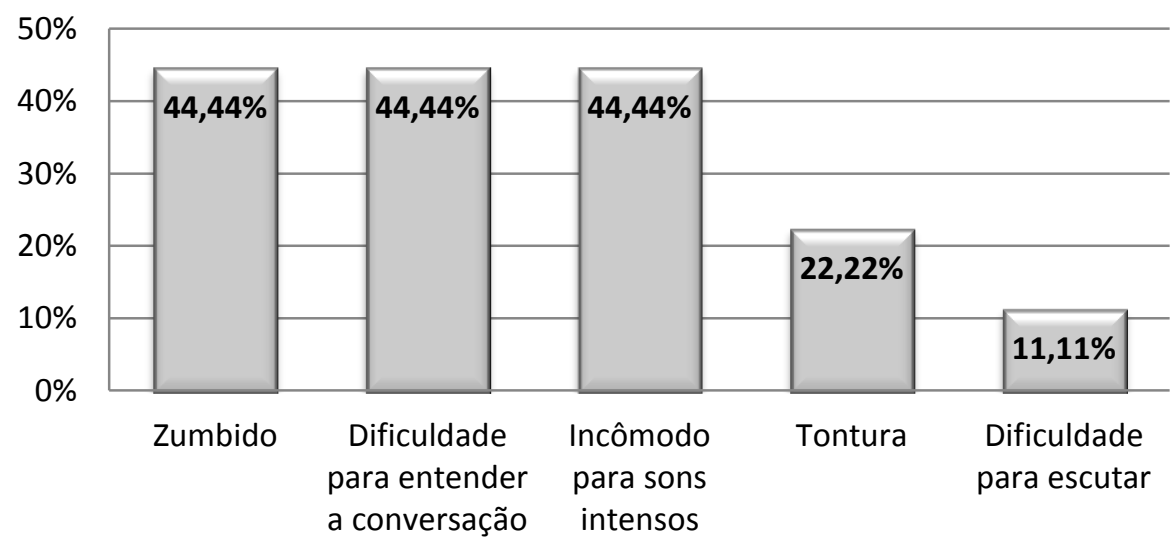

Figura 1. Sintomas auditivos relatados pelos participantes do estudo.

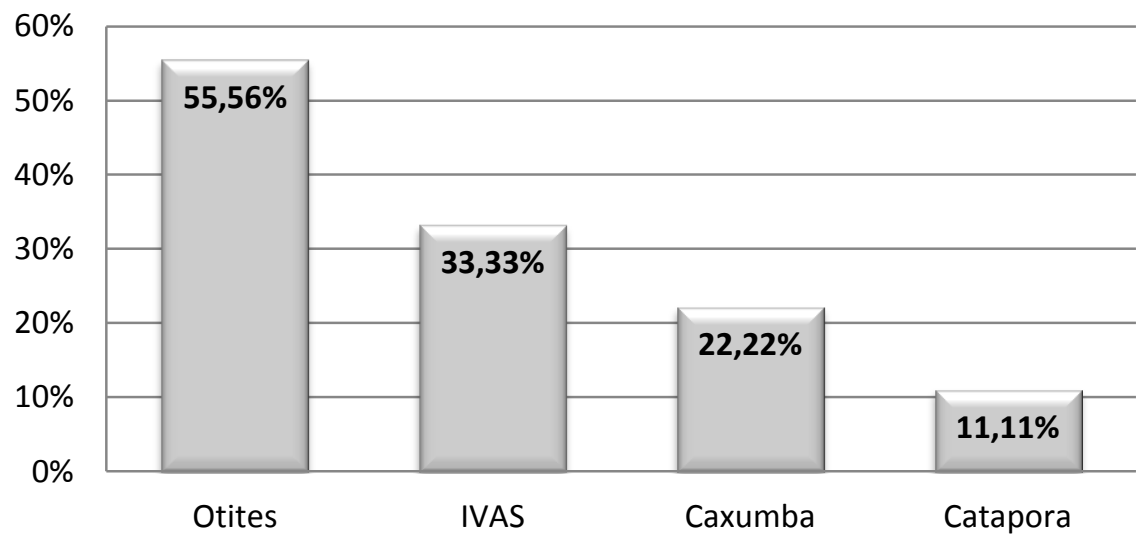

Figura 2. Doenças pregressas relatadas pelos participantes do estudo.

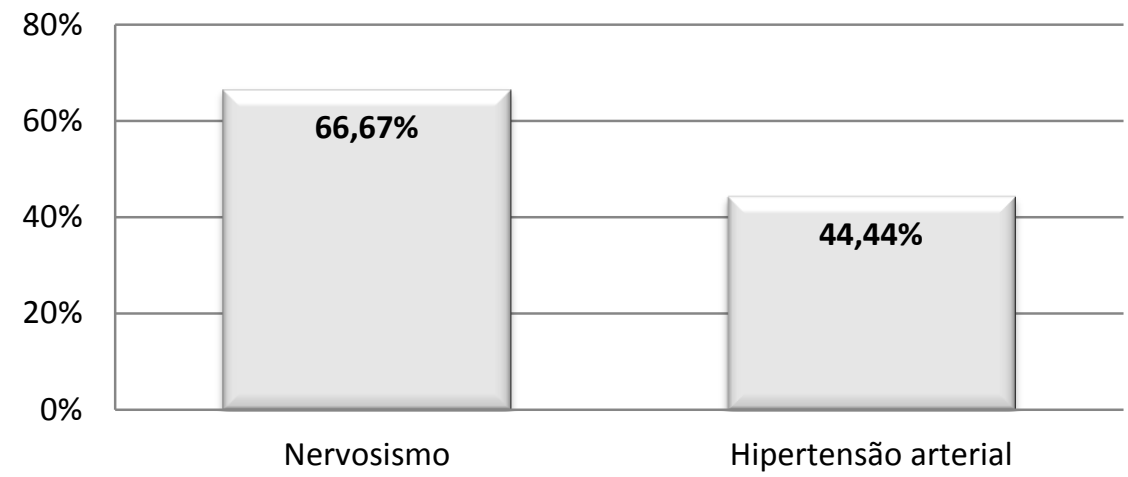

Figura 3. Sintomas extra-auditivos relatados pelos participantes do estudo. 
Quanto ao uso de medicamentos, seis (66,67\%) participantes referiram fazer uso habitual de medicação, sendo que quatro $(44,44 \%)$ participantes referiram fazer uso de medicamentos para controle da pressão arterial, um $(11,11 \%)$ participante referiu uso contínuo de "Fenobarbital" e um (11,11\%) referiu uso constante de medicação para problemas estomacais.

Em relação ao histórico de cirurgia, cinco $(55,56 \%)$ referiram já terem se submetido há pelo menos um procedimento cirúrgico, sendo: vasectomia, retirada do apêndice e da vesícula, correção ortopédica por fratura na perna, retirada de tumor no braço e hérnia.

Quanto ao uso de álcool e fumo, seis $(66,67 \%)$ referiram fazer uso de tabaco e dois $(22,22 \%)$ referiram fazer uso de álcool.

Em relação aos sintomas extra-auditivos, o nervosismo foi o sintoma mais relatado pelos participantes do estudo, como mostra a Figura 3.

No que se refere à exposição aos químicos, cinco $(55,56 \%)$ participantes referiram ter apresentado exposição constante a combustíveis, tais como o diesel, a gasolina e o etanol. Esse relato não foi somente pela prática atual da profissão, mas também por já terem exercido outras práticas profissionais relacionadas à exposição ao agente químico.

Após aplicação da entrevista fonoaudiolígica com a finalidade de caracterizar os sintomas auditivos e extra-auditivos, foi realizada a inspeção do canal auditivo externo, não sendo visualizado nenhum impedimento para a realização da avaliação audiológica básica, constituída pela pesquisa dos limiares tonais, vocais e imitanciometria.

Pela pesquisa dos limiares tonais foi possível caracterizar o perfil auditivo dos motoristas de caminhão quanto ao grau, tipo de perda auditiva, traçado audiométrico e lateralidade. A análise se deu pela descrição do perfil auditivo, levando em consideração as orelhas direita e esquerda, quanto ao grau e tipo de perda auditiva.

Em relação ao grau de perda auditiva, baseada na classificação de Lloyd e Kaplan ${ }^{6}$, que determina o grau da perda pela média das frequências de $500 \mathrm{~Hz}, 1000 \mathrm{~Hz}$ e $2000 \mathrm{~Hz}$, notase na Tabela 1 que a maioria dos participantes apresentou média tritonal dentro dos padrões da normalidade. $O$ participante que apresentou alteração na média tritonal é possível observar que na orelha direita o grau apresentado foi severo e na orelha esquerda foi moderadamente severo.

Quanto ao tipo de perda auditiva, baseada na classificação de Silman e Silverman ${ }^{7}$, observa-se na Tabela 2 que a maioria dos participantes não apresentou perda auditiva, visto que os limiares tonais apresentaram-se até 25 dBNA (decibel em nível de audição). Os participantes que apresentaram perda auditiva, o tipo neurossensorial foi mais evidente, tanto na orelha direita quanto na orelha esquerda.

$\mathrm{Na}$ Tabela 3 são apresentadas alterações nos limiares auditivos dos participantes do estudo, sendo mais evidente na orelha direita.

Tabela 1. Perfil auditivo dos participantes do estudo quanto ao grau da perda auditiva, baseada na média tritonal $(500 \mathrm{~Hz}, 1000 \mathrm{~Hz}, 2000 \mathrm{~Hz})^{6}$.

\begin{tabular}{lcccccc}
\hline Grau de perda auditiva & \multicolumn{2}{c}{ Orelha direita } & \multicolumn{2}{c}{ Orelha esquerda } & \multicolumn{2}{c}{ Total } \\
\cline { 2 - 7 } & $\mathbf{N}$ & $\mathbf{\%}$ & $\mathbf{N}$ & $\mathbf{\%}$ & $\mathbf{N}$ & $\%$ \\
\hline Normal & 8 & 88,89 & 8 & 88,89 & 16 & 88,88 \\
Leve & 0 & 0,0 & 0 & 0,0 & 0 & 0,0 \\
Moderado & 0 & 0,0 & 0 & 0,0 & 0 & 0,0 \\
Moderadamente Severo & 0 & 0,0 & 1 & 11,11 & 1 & 5,56 \\
Severo & 1 & 11,11 & 0 & 0,0 & 1 & 5,56 \\
Profundo & 0 & 0,0 & 0 & 0,0 & 0 & 0,0 \\
Total & 9 & 100 & 9 & 100 & 18 & 100 \\
\hline
\end{tabular}

$\mathrm{p}=0,735$ (Teste $\mathrm{G}$ ) 
Tabela 2. Perfil auditivo dos participantes do estudo quanto ao tipo de perda auditiva ${ }^{7}$.

\begin{tabular}{lcccccc}
\hline Tipo de perda & \multicolumn{2}{c}{ Orelha direita } & \multicolumn{2}{c}{ Orelha esquerda } & \multicolumn{2}{c}{ Total } \\
\cline { 2 - 7 } auditiva & $\mathbf{N}$ & $\mathbf{\%}$ & $\mathbf{N}$ & $\mathbf{\%}$ & $\mathbf{N}$ & $\%$ \\
\hline Normal & 5 & 55,56 & 6 & 66,67 & 11 & 61,11 \\
Condutivo & 0 & 0,0 & 0 & 0,0 & 0 & 0,0 \\
Misto & 1 & 11,11 & 1 & 11,11 & 2 & 11,11 \\
Neurossensorial & 3 & 33,33 & 2 & 22,22 & 5 & 27,78 \\
Total & 9 & 100 & 9 & 100 & 18 & 100 \\
\hline p $=0,9615$ (Teste G) & & & & & &
\end{tabular}

Tabela 3. Apresentação dos participantes com e sem alteração nos limiares tonais auditivos.

\begin{tabular}{lcccc}
\hline \multirow{2}{*}{ Condição } & \multicolumn{2}{c}{ Orelha direita } & \multicolumn{2}{c}{ Orelha esquerda } \\
\cline { 2 - 5 } & $\mathbf{N}$ & $\%$ & $\mathbf{N}$ & $\%$ \\
\hline Sem alteração & 5 & 55,56 & 6 & 66,67 \\
Com alteração & 4 & 44,44 & 3 & 33,33 \\
Total & 9 & 100 & 9 & 100 \\
\hline p=0,50 (Teste exato de Fisher) & & &
\end{tabular}

Entre os participantes que apresentaram acometidas na orelha direita e duas na orelha alterações nos limiares auditivos, três alterações esquerda são sugestivas de PAIR (Tabela 4).

Tabela 4. Ocorrência das alterações auditivas sugestivas de Perda Auditiva Induzida por Ruído.

\begin{tabular}{lcccccc}
\hline Sugestivo & \multicolumn{2}{c}{ Orelha direita } & \multicolumn{2}{c}{ Orelha esquerda } & \multicolumn{2}{c}{ Total } \\
\cline { 2 - 7 } de PAIR & $\mathbf{N}$ & $\mathbf{\%}$ & $\mathbf{N}$ & $\mathbf{\%}$ & $\mathbf{N}$ & $\%$ \\
\hline Sim & 3 & 33,33 & 2 & 22,22 & 5 & 27,78 \\
Não & 6 & 66,67 & 7 & 77,78 & 13 & 72,22 \\
Total & 9 & 100 & 9 & 100 & 18 & 100 \\
\hline p=0,6471 (Teste exato de Fisher) & & & &
\end{tabular}

Ao descrever o perfil auditivo dos participantes do estudo quanto aos achados timpanométricos, a Tabela 5 mostra que a maioria dos participantes apresentou normalidade timpanométrica.
Quanto aos achados do reflexo acústico, os resultados evidenciaram maiores alterações dos limiares na orelha direita, como mostra a Tabela 6.

Tabela 5. Perfil auditivo dos participantes do estudo quanto aos achados timpanométricos.

\begin{tabular}{lcccccc}
\hline \multirow{2}{*}{ Timpanogramas } & \multicolumn{2}{c}{ Orelha direita } & \multicolumn{2}{c}{ Orelha esquerda } & \multicolumn{2}{c}{ Total } \\
\cline { 2 - 7 } & $\mathbf{N}$ & $\mathbf{\%}$ & $\mathbf{N}$ & $\mathbf{0}$ & $\mathbf{N}$ & $\%$ \\
\hline Tipo A & 8 & 88,89 & 7 & 77,78 & 15 & 83,33 \\
Tipo B & 0 & 0,0 & 0 & 0,0 & 0 & 0,0 \\
Tipo C & 0 & 0,0 & 1 & 11,11 & 1 & 5,56 \\
Tipo Ar & 0 & 0,0 & 0 & 0,0 & 0 & 0,0 \\
Tipo Ad & 1 & 11,11 & 1 & 11,11 & 2 & 11,11 \\
Total & 9 & 100 & 9 & 100 & 18 & 100 \\
\hline p=0,8349 (Teste G) & & & & & &
\end{tabular}


Tabela 6. Perfil auditivo dos participantes do estudo quanto aos achados do reflexo acústico.

\begin{tabular}{lcccccc}
\hline \multirow{2}{*}{ Reflexo acústico } & \multicolumn{2}{c}{ Orelha direita } & \multicolumn{2}{c}{ Orelha esquerda } & \multicolumn{2}{c}{ Total } \\
\cline { 2 - 7 } & $\mathbf{N}$ & $\mathbf{\%}$ & $\mathbf{N}$ & $\mathbf{\%}$ & $\mathbf{N}$ & $\%$ \\
\hline Presentes e normais & 3 & 33,33 & 6 & 66,67 & 9 & 50 \\
Presentes e elevados & 3 & 33,33 & 1 & 11,11 & 4 & 22,22 \\
Presentes e diminuídos & 1 & 11,11 & 0 & 0,0 & 1 & 5,56 \\
Ausentes & 2 & 22,22 & 2 & 22,22 & 4 & 22,22 \\
Total & 9 & 100 & 9 & 100 & 18 & 100 \\
\hline \multicolumn{1}{c}{$\mathrm{p}=0,3270$ (Teste G) } & & & & & &
\end{tabular}

\section{DISCUSSÃO}

Os caminhoneiros são considerados um dos principais agentes do setor de transporte devido ao fato desta classe de trabalhadores movimentarem efetivamente o sistema econômico do país.

Por estarem expostos a elevados níveis de pressão sonora produzidos pelo motor do caminhão a investigação dos achados audiológicos nesta classe de trabalhadores, bem como verificar a ocorrência de perdas auditivas induzidas pelo ruído e os sintomas auditivos e extra-auditivos faz-se necessário.

Trata-se de um estudo com uma amostra reduzida, pois apenas nove caminhoneiros aceitaram participar do estudo. A princípio, estimava-se uma amostra de 40 caminhoneiros usuários da empresa de Serviço Social do Transporte - Serviço Nacional de Aprendizagem do Transporte (SEST-SENAT), onde todos foram devidamente convidados mediante listagem disponibilizada pela responsável do setor de recursos humanos. Entretanto, como a participação é de livre consentimento do participante, apenas um compareceu para realização dos exames auditivos, sendo necessário convidar motoristas autônomos de conhecimento das pesquisadoras para a composição da amostra.

Com intuito em caracterizar a amostra quanto ao sexo, o estudo evidenciou que todos os participantes eram do sexo masculino. Essa ocorrência se deu pelo fato da maior inserção dos homens nessa prática profissional. Quanto à idade, a maioria dos participantes apresentou idades mais avançadas, o que poderia ter levado à observação de alterações auditivas decorrentes ao avanço da idade.

Com relação às queixas auditivas, o zumbido, a dificuldade para entender a conversação e o incômodo a sons intensos foram as mais relatadas pela população estudada.
Os indivíduos que estão expostos a ruídos intensos podem apresentam além de uma perda auditiva, sintomas auditivos como zumbido e dificuldade para compreender a fala, além de sintomas vestibulares, digestivos e comportamentais ${ }^{7}$.

O zumbido é considerado um dos sintomas mais relatados por populações expostas ao ruído ${ }^{8}$. Com a finalidade de estudar as características do zumbido em trabalhadores expostos ao ruído, autores observaram que $22 \%$ do total da amostra relataram zumbido, sendo este relato maior no sexo masculino e em trabalhadores com tempo médio de atividade ocupacional de 6,8 anos. Em trabalhadores com audição normal, o zumbido esteve presente em $71 \%$ da amostra.

Quanto à dificuldade para entender a conversação, autor ${ }^{9}$ relatou que elevados níveis de pressão sonora pode causar transtornos na comunicação, pois o ruído pode mascarar a voz, prejudicando assim a compreensão da fala.

A dificuldade para entender a conversação caracteriza-se em uma desvantagem psicossocial, podendo ser observada em populações expostas ao ruído com ou sem perda auditiva ${ }^{10}$. A mesma observação foi feita pelo autor quanto ao incômodo a sons intensos. Tais queixas podem influenciar negativamente no ambiente social e de trabalho do indivíduo.

As condições de saúde em geral e os hábitos nocivos também podem influenciar no funcionamento do sistema auditivo.

Autores $^{11}$ referiram que a perda auditiva induzida pelo ruído pode ser desencadeada ou agravada pelo tabagismo, ruído, vibração e contaminantes químicos. De acordo com estudo, - monóxido de carbono presente nos componentes do cigarro resulta na redução dos níveis de oxigênio na cóclea, levando a vasoconstrição, aumento do fluxo sanguíneo e diminuição do transporte de oxigênio, o que 
contribuirá no aparecimento de perdas auditivas, principalmente na região basal da cóclea ${ }^{11}$.

Em outro estudo, os autores referiram que além da perda auditiva a nicotina pode agravar o zumbido pelo fato de serem estimulantes e por provocarem contrições dos vasos sanguíneos. A exposição ao ruído associado ao hábito de fumar e o fator da idade podem ser grandes fatores de risco para a perda auditiva, aumentando assim, os sintomas auditivos e agravando o aparecimento da $\mathrm{PAIR}^{12}$. No presente estudo pode-se observar que $44,44 \%$ dos caminhoneiros apresentam sintoma de zumbido, sendo que a maioria é tabagista e apresentam idade média de 44,22 anos.

Dentre os sintomas extra-auditivos, notase no presente estudo que o nervosismo foi o sintoma mais relatado pelos caminhoneiros.

Outros autores ${ }^{13}$ relataram que 0 nervosismo é um sintoma comum em trabalhadores expostos a ruído. Com intuito em investigar as queixas de saúde e os achados audiológicos em duas classes de trabalhadores expostos a ruído e vibração, os autores observaram que a queixa mais frequentes foi o nervosismo. A mesma observação foi feita pelos autores do estudo quanto às queixas de hipertensão arterial, corroborando com os achados do presente estudo.

Além do ruído, a exposição a químicos também pode influenciar negativamente no funcionamento do sistema auditivo, sendo potencializada se a exposição for combinada.

A perda auditiva de origem ocupacional vem representando um agravante problema na saúde pública. No ambiente de trabalhado existem diversos riscos à saúde, além do ruído que podem gerar efeitos na audição do trabalhador, pode ser citada também a exposição a químicos, o uso de medicamentos e as vibrações $^{14}$. No presente estudo pode-se observar que além da exposição do ruído, 55,56\% estão expostos ou já foram expostos em outros locais de trabalho a produtos químicos, além de fazer uso de medicações contínuas.

Os solventes orgânicos são substâncias químicas que altera tanto o funcionamento do sistema auditivo quanto o sistema vestibular. Há maiores efeitos no sistema auditivo central, causando danos no tronco encefálico, no cerebelo e no córtex auditivo. Por esse motivo, o solvente orgânico, composto químico presente em vários tipos de combustíveis, pode afetar o sistema auditivo central que comanda o reflexo acústico devido aos efeitos sinérgicos de uma coexposição a ruído e solventes ${ }^{15}$.

Ao verificar o perfil auditivo dos caminheiros do presente estudo quanto ao grau de perda auditiva é possível observar que a maioria apresentou média dos limiares auditivos nas frequências de 500, 1000 e $2000 \mathrm{~Hz}$ dentro dos padrões da normalidade. Entretanto, sabe-se que a exposição a elevados níveis de pressão sonora afetam limiares de audibilidade em frequências mais altas, ou seja, as frequências de 3000,4000 e $6000 \mathrm{~Hz}$.

Outros autores ${ }^{16}$ relataram que 0 acometimento inicial da perda auditiva induzida pelo ruído se dá nas frequências, pela ordem que se segue, de 6000,4000 e $3000 \mathrm{~Hz}$, progredindo mediante a persistência da exposição para as frequências de 250, 500, 1000, 2000 e $8000 \mathrm{~Hz}$.

$\mathrm{Em}$ outro estudo foi observado que a perda auditiva induzida por ruído é de caráter irreversível, do tipo neurossensorial, simétrica e com presença de entalhe, cuja alteração acomete as estruturas internas da cóclea ${ }^{17}$. Tal alteração também pode ser observada no presente estudo, visto que os participantes que apresentaram alterações auditivas com características audiológicas sugestivas de uma perda auditiva induzida por ruído, sendo a orelha direita a mais acometida.

Um estudo feito no Canadá objetivou medir o nível de pressão sonora produzida por caminhões. Foram realizadas 400 medições e observado que o motorista ao dirigir o caminhão com as janelas fechadas e rádio desligado o nível equivalente (Leq) de pressão sonora presente em suas atividades foi de 78 a $89 \mathrm{~dB}$ (A). Um aumento de 3,9 dB (A) foi observado ao dirigir o caminhão com as janelas abertas e o rádio ligado ${ }^{4}$.

Quanto aos achados timpanométricos a maioria dos participantes apresentou normalidade do sistema tímpano-ossicular. Esse achado pode ser justificado pelo fato do ruído contínuo afetar a orelha interna, e não a orelha média.

Estudo aponta que as células ciliadas externa do órgão de Corti são as primeiras estruturas a sofrerem danos causados pelo ruído devido ao fato destas estruturas serem mais vulneráveis mediante exposição ao ruído ${ }^{18}$.

O ruído somente afetará o sistema tímpano-ossicular se este for de impacto, o que não ocorre mediante exposição ao ruído produzido pelos motores de caminhões. 
As ocorrências de alterações de orelha média observados no presente estudo são de outros fatores causais, tais como a disjunção de cadeia ossicular e alteração no funcionamento da tuba auditiva. Essas alterações não são justificadas pela exposição contínua a elevados níveis de pressão sonora.

Quanto aos achados dos limiares do reflexo acústico observa-se no presente estudo que a maioria apresentou alteração, sendo a orelha direita com maior representatividade na alteração. Porém, a análise estatística revelou não haver relação quanto à lateralidade.

Um estudo realizado em 2008 com intuito de investigar o reflexo acústico em trabalhadores expostos a elevados níveis de pressão sonora e compará-los com o grupo controle revelou haver diferença significante entre os grupos estudados, ou seja, o grupo de trabalhadores expostos ao ruído apresentou um incremento no limiar do reflexo acústico, sendo que quanto maior o tempo de exposição maior o limiar do reflexo acústico. Assim, os autores do estudo concluíram que é promissora a pesquisa dos limiares do reflexo acústico em populações expostas a ruído, pois apresenta um grande potencial no diagnóstico precoce da perda auditiva induzida por ruído, tendo uma vantagem de identificar os efeitos do ruído antes mesmo da alteração auditiva neurossensorial, e ainda, de forma objetiva $^{19}$.

Apesar dos participantes do estudo apresentarem fatores de risco para perda auditiva, como hábitos nocivos à saúde (fumo e álcool), idade mais avançada, sintomas auditivos e extra-auditivos, uso de medicações e exposições a produtos químicos não foram observados valores significantes quanto aos achados audiológicos. Tal achado pode ser justificada pelo tamanho da amostra do estudo.

Não possível fazer maiores inferências sobre os achados audiológicos devido ao número restrito de participantes. Entretanto, é possível observar maior ocorrência de sintomas auditivos e extra-auditivos nesta classe de trabalhadores em relação à ocorrência de perda auditiva induzida por ruído.

Assim, acredita-se ser necessário desenvolver ações de prevenção a essa classe de trabalhadores visando melhores condições de trabalho, tais como: ações ambientais e em conjunto para que ocorra a manutenção periódica dos veículos, medidas para reduzir o nível de pressão sonora dentro das cabines dos caminhões, pausas mais frequentes em suas jornadas de trabalho, além de promoção à saúde, visando melhor qualidade de vida e desempenho em suas práticas profissionais.

Ainda, recomenda-se novos estudos com a mesma classe de trabalhadores e em amostras com maior representatividade, uma vez que há um carência de estudos publicados sobre a audição dos caminhoneiros.

\section{CONFLITOS DE INTERESSE}

Os autores declaram não haver qualquer potencial de conflito de interesse que possa interferir na imparcialidade deste trabalho científico.

\section{REFERÊNCIAS}

1. Botelho LJ, Diesel LE, Adamczyk JF, Zeferino MT. Profissão motorista de caminhão: uma visão (im)parcial. Sau Transf Soc. 2011;2(1):108-13.

2. Masson VA, Monteiro MI. Estilo de vida, aspectos de saúde e trabalho de motoristas de caminhão. Rev Bras Enferm. 2010;63(4):533-40. DOI: http://dx.doi.org/10.1590/S0034-

\section{6}

3. Lopes AC, Otowiz VG, Lopes PMB, Lauris JRP, Santos CC. Prevalência de perda auditiva induzida por ruído em motoristas. Int Arch Otorhinolaryngol. 2012;16(4):509-14. DOI:

http://dx.doi.org/10.7162/S1809-

\section{3}

4. Lopes G, Russo ICP, Fiorini AC. Estudos da audição e da qualidade de vida em motoristas de caminhão. Rev CEFAC. 2007;9(4):532-42. DOI: http://dx.doi.org/10.1590/S151618462007000400014

5. Guerra. MR, Lourenço PMC, Bustamante-Teixeira MT, Alves MJM. Prevalência de perda auditiva induzida por ruído em empresa metalúrgica. Rev Saúde Pública. 2005;39(2):238-44. DOI: http://dx.doi.org/10.1590/S0034-

\section{5}

6. Conselho Federal e Regional de Fonoaudiologia. Manual de procedimentos em audiometria tonal, logoaudiometria e medidas de imitância acústica. 2013 [acesso em 9 mar 2016]. Disponível em: http://www.fonoaudiologia.org.br/publicacoes/Manu al\%20de\%20Audiologia.pdf

7. Costa JB, Rosa SAB, Borges LL, Camarano MRH. Caracterização do perfil audiológico em trabalhadores expostos a ruídos ocupacionais. Estudos. 2015;42(3):273-87.

8. Steinmetz LG, Zeigelboim BS, Lacerda AB, Morata TC, Marques JM. Características do zumbido em 
trabalhadores expostos a ruído. Rev Bras Otorrinolaringol. 2009;75(1):7-14. DOI: http://dx.doi.org/10.1590/S0034-

\section{2}

9. Medeiros LB. Ruído: efeitos extra-auditivos no corpo humano [monografia]. Porto alegre: Centro de Especialização em Fonoaudiologia Clínica: Audiologia Clínica; 1999. [acesso em 9 mar 2016]. Disponível em: http://www.cefac.br/library/teses/3f1dbb59a55ef633 5162f736db63c961.pdf

10. Campelo LMP. Identificação de sintomas auditivos e extra-auditivos em trabalhadores expostos a níveis elevados de pressão sonora e sua relação com o tempo de exposição [dissertação]. Rio de Janeiro: Universidade Veiga de Almeida; 2007. [acesso em 9 mar 2016]. Disponível em: http://www.uva.br/mestrado/dissertacoes fonoaudio logia/liziane-maria-campelo-identificacao-desintomas-auditivos-e-extra-auditivos-em-trabal.pdf 11. Ferreira DG, Oliveira GL, Meira AL, Lacerda A. Efeitos auditivos da exposição combinada: interação entre monóxido de carbono, ruído e tabagismo. Rev Soc Bras Fonoaudiol. 2012;12(4):405-11. DOI: http://dx.doi.org/10.1590/S1516-

\section{7}

12. Lacerda A, Figueiredo G, Neto JM, Marques JM. Achados audiológicos e queixas relacionadas à audição dos motoristas de ônibus urbano. Rev Soc Bras Fonoaudiol. 2010;15(2):161-6. DOI: http://dx.doi.org/10.1590/S1516-

\section{3}

13. Fernandes M, Morata TC. Estudo dos efeitos auditivos e extra-auditivos na exposição ocupacional a ruído e vibração. Rev Bras Otorrinolaringol. 2002;68(5):705-13. DOI:

http://dx.doi.org/10.1590/S0034-

\section{7}

14. Alves AS, Fiorini AC. A autopercepção do handicap auditivo em trabalhadores de uma indústria têxtil. Distúrb Comum. 2012;24(3):337-49.

15. Tochetto TM, Quevedo LS, Siqueira MA. Condição auditiva de frentistas. Rev CEFAC. 2012;15(5):1137-47. DOI: $\quad$ http://dx.doi.org/10.1590/S151618462012005000107

16. Guida HL, Morini RG, Cardoso ACV. Avaliação audiológica e de emissões otoacústicas em indivíduos expostos a ruído e praguicidas. Int Arch Otorhinolaryngol. 2009;13(3):264-9.

17. Caldart AU, Adriano CF, Terruel I, Martins RF, Caldart AU, Mocellin M. A prevalência da Perda Auditiva Induzida pelo Ruído em Trabalhadores de Indústria Têxtil. Int Arch Otorhinolaryngol. 2006;10(3):192-6.

18. Brito VPS. Incidência de perda auditiva induzida por ruído em trabalhadores de uma fábrica [monografia]. Goiânia: Centro de Especialização em Fonoaudiologia Clínica: Audiologia Clínica; 1999. [acesso em 9 mar 2016]. Disponível em: http://www.cefac.br/library/teses/a2eff1432f7ff2a4e d0cc9f34fc1e5d6.pdf

19. Gomes FLT. Estudo do efeito da exposição ao ruído sobre o limiar do reflexo estapédico [dissertação]. Rio de Janeiro: Universidade Veiga de Almeida; 2008. [acesso em 9 mar 2016]. Disponível em: http://www.uva.br/mestrado/dissertacoes fonoaudio logia/fernanda lucia.pdf

Recebido para publicação em 03/12/2015

Revisado em 03/06/2016

Aceito em 22/09/2016 